\title{
A Graph-with-Loop Structure for a Topological Representation of 3D Objects ${ }^{\star}$
}

\author{
Rocio Gonzalez-Diaz, María José Jiménez, Belen Medrano*^, and Pedro Real \\ Applied Math Department, University of Seville, Spain \\ \{rogodi,majiro, belenmg, real\}@us.es \\ http://alojamientos.us.es/gtocoma
}

\begin{abstract}
Given a cell complex $K$ whose geometric realization $|K|$ is embedded in $\mathbf{R}^{3}$ and a continuous function $h:|K| \rightarrow \mathbf{R}$ (called the height function), we construct a graph $G_{h}(K)$ which is an extension of the Reeb graph $R_{h}(|K|)$. More concretely, the graph $G_{h}(K)$ without loops is a subdivision of $R_{h}(|K|)$. The most important difference between the graphs $G_{h}(K)$ and $R_{h}(|K|)$ is that $G_{h}(K)$ preserves not only the number of connected components but also the number of "tunnels" (the homology generators of dimension 1 ) of $K$. The latter is not true in general for $R_{h}(|K|)$. Moreover, we construct a map $\psi: G_{h}(K) \rightarrow K$ identifying representative cycles of the tunnels in $K$ with the ones in $G_{h}(K)$ in the way that if $e$ is a loop in $G_{h}(K)$, then $\psi(e)$ is a cycle in $K$ such that all the points in $|\psi(e)|$ belong to the same level set in $|K|$.
\end{abstract}

\section{Reeb Graphs and Tunnels}

We are interested in analyzing and visualizing intrinsic properties of geometric models and scientific data. Specifically, Reeb graphs [13], which express the connectivity of level sets, have been used in the past to construct data structures and user-interfaces for modeling and visualization applications [5].

Let $X$ be a topological space and $h: X \rightarrow \mathbf{R}$ a continuos map. A level set is the primage of a constant value, $h^{-1}(t)$. Call a connected component of a level set a contour. Two points $x, y \in X$ are equivalent, $x \sim y$, if they belong to the same contour, that is, if $h(x)=h(y)$ and $x$ and $y$ are connected by a path on $X$. The Reeb graph of $h, R_{h}(X)$, is the quotient space defined by this equivalence relation. Observe that, by construction, the Reeb graph has a point for each contour and the connection is provided by $\psi: X \rightarrow R_{h}(X)$ that maps each point $x$ to its equivalence class. Even though the Reeb graph loses a lot of the original topological structure, some things can be said: a tunnel in $X$ that maps (by $\psi$ ) to a tunnel in $R_{h}(X)$ cannot be continuously deformed to a single point, and two tunnels in $X$ that map to different tunnels in $R_{h}(X)$. The number of connected components of $X, \beta_{0}(X)$, is preserved and the number of tunnels of $X, \beta_{1}(X)$, cannot increase, i.e. $\beta_{0}\left(R_{h}(X)\right)=\beta_{0}(X)$ and $\beta_{1}\left(R_{h}(X)\right) \leq \beta_{1}(X)$.

\footnotetext{
* Partially supported by Junta de Andalucía (FQM-296 and TIC-02268) and Spanish Ministry for Science and Education (MTM-2006-03722).

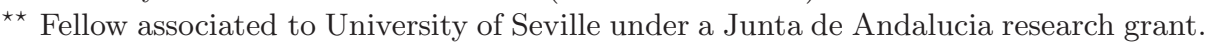




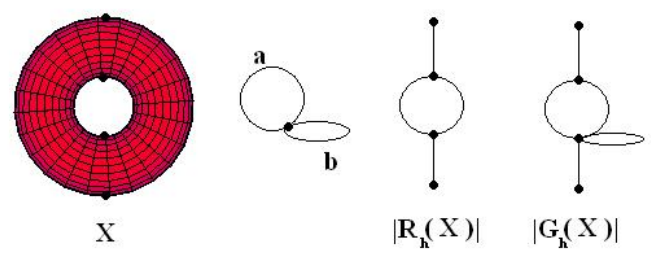

Fig. 1. From left to right, the torus $X$; representative cycles of the two tunnels of $X$ (the tunnel $a$ is obvious and the other one, $b$, is not); a geometric realization of the Reeb Graph $R_{h}(X)$ that $h$ associates to each point on $X$ its elevation; and a geometric realization of the graph with loops $G_{h}(X)$

In 2] the authors adapt concepts developed for smooth manifolds to discrete surface models, introducing an extended Reeb graph representation for a generic polyhedral surface. Their approach is based on the computation of a sufficiently dense number of contour lines and the definition of the Reeb graph from the contour set. However, such a construction is actually not an extension of the Reeb graph itself, but rather an application of its definition in the discrete domain. In [4, tight upper and lower bounds of the number of tunnels in the Reeb graph that depend on the genus, the number of boundary components and whether or not the 2-manifold is orientable, is given.

In this paper, we focus on objects embedded in $\mathbf{R}^{3}$. Several combinatorial structures may represent a cellular subdivision which models an object such as simplicial, cubical and simploidal complexes. Roughly speaking, the cells of a given simplicial complex are simplices (vertices, edges, triangles and tetrahedra); vertices, edges, squares and cubes constitute the collection of cells of a cubical complex; in the case of simploidal complexes, which generalize both simplicial and cubical complexes (see [3]), cells are cartesian products of simplices. In all the cases they fit together in a natural way to form the object (see [113]).

From now on, a graph $G=(V, E)$, where $V$ is a set of vertices and $E$ a set of edges, is considered as a particular complex only with vertices and edges. Given

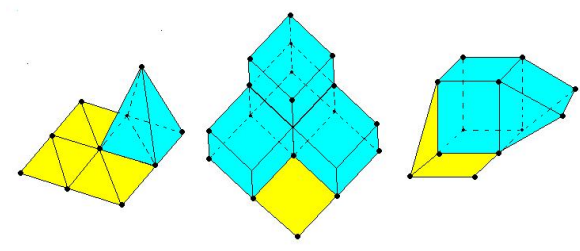

Fig. 2. A simplicial, cubical and simploidal complex

a complex $K$ (simplicial, cubical or simploidal), a geometric realization of it (e.g. a 3D triangulated surface) $|K|$, and a continuous function $h:|K| \rightarrow \mathbf{R}$, our aim is the computation of a graph $G_{h}(K)$ and a function $\Psi: G_{h}(K) \rightarrow K$ with the following properties: 
- $G_{h}(K)$ has the same number of connected components and tunnels than $K$.

- Each loop (an edge such that its endvertices are the same) of $G_{h}(K)$ maps to a non-contractible cycle $c$ in $K$ such that $|c|$ (the geometric realization of all the cells in $c$ ) lies in a contour of $|K|$, two loops in $G_{h}(K)$ map to non-homologous cycles in $K$, and each edge in $G_{h}(K)$ map to a path in $K$.

- If we do not consider the loops in $G_{h}(K)$, then $G_{h}(K)$ is a subdivision of the Reeb graph $R_{h}(K)$.

Therefore, $G_{h}(K)$ can be seen as an extension of $R_{h}(K)$ such that not only the number of connected components and tunnels of $G_{h}(K)$ and $K$ coincide (this is not true, in general, for $R_{h}(K)$ ), but also there exists a one-by-one identification of tunnels in $G_{h}(K)$ with tunnels in $K$ in the way that if $e$ is a loop in $G_{h}(K)$, then $\psi(e)$ is a cycle in $K$ such that all the points in $|\psi(e)|$ have the same height.

\section{Algebraic-Topological Models for 3D Objects}

This section introduces the algebraic topology background needed to understand the rest of the paper, which is essentially extracted from Munkres' book [12. The concept of AT-model established in 8 89 for cohomology computation of $3 \mathrm{D}$ digital images is adapted here to solve the problem of computing the graph $G_{h}(K)$ and the function $\Psi: G_{h}(K) \rightarrow K$. Without lost of generality, we will consider that the ground ring is $\mathbf{Z} / 2$.

A chain complex $\mathcal{C}$ is a sequence $\left\{C_{q}, d_{q}\right\}$ of abelian groups $C_{q}$ and homomorphisms $d_{q}: C_{q+1} \rightarrow C_{q}$, such that, for all $q, d_{q} d_{q+1}=0$. The set $\left\{d_{q}\right\}_{q \geq 0}$ is called the differential of $\mathcal{C}$. The chain complex $\mathcal{C}$ is free if $C_{q}$ is a free abelian group for each $q$; it is finite if there exists an integer $n>0$ such that $C_{q}=0$ for $q>n$ and each abelian group $C_{q}$ is finitely generated. All chain complexes considered here are finite and free. A chain $c$ in $\mathcal{C}$ is a $q$-cycle if $c \in \operatorname{Ker} d_{q}$. If $c \in \operatorname{Im} d_{q+1}$ then $a$ is called a $q$-boundary. Denote the groups of $q$-cycles and $q$-boundaries by $Z_{q}$ and $B_{q}$ respectively. Define the integer $q$ th homology group to be the quotient group $Z_{q} / B_{q}$, denoted by $H_{q}(\mathcal{C})$. We say that $c$ is a representative $q-c y c l e$ of the homology generator $c+B_{q}$ (denoted by $[c]$ ). For each $q$, the $q$ th homology group $H_{q}(\mathcal{C})$ is a finitely generated free abelian group. The rank of $H_{q}$, denoted by $\beta_{q}$, is called the $q$ th Betti number of $\mathcal{C}$. Homology is a powerful topological invariant, which characterizes an object by its $q$-dimensional "holes" (connected components, tunnels and cavities).

Let $K$ be a complex (simplicial, cubical or simploidal). A $q$-chain $c$ is a formal sum of $q$-cells (where $q$ is the dimension of the cell) in $K$. Let $\left\{\sigma_{1}^{q}, \ldots, \sigma_{m_{i}}^{q}\right\}$ be the set of $q$-cells in $K$, then $c=\sum_{i=1}^{m_{i}} \lambda_{i} \sigma_{i}^{q}$, where $\lambda_{i} \in\{0,1\}$. Alternatively, we can think of $c$ as the set $\left\{\sigma_{i}^{q}\right.$, such that $\left.\lambda_{i}=1\right\}$, and the sum of two $q$-chains as their symmetric difference. The $q$-chains together with the addition operation form the group of $q$-chains denoted as $C_{q}(K)$. The differential of a $q$-cell $\sigma$ in $K, d_{q}(\sigma)$, is the sum of the $(q-1)$-cells in $K$ that belong to the boundary of $\sigma$. By linearity, the differential can be extended to $q$-chains. The chain complex $C(K)$ is the sequence of chain groups $C_{q}(K)$ connected by the homomorphisms $d_{q}$. The homology of $K$ is defined as the homology of $C(K)$. Since we work with 
objects embedded in $\mathbf{R}^{3}$, the homology groups are torsion-free (see [1, ch.10]). Moreover, Theorem of Universal Coefficient [12] ensures that all the homology information can be computed working with coefficients in $\mathbf{Z} / 2$.

An AT-model for $K$ is established in 89 and used to obtain the homology and representative cycles of homology generators of $K$. An AT-model can be computed starting from an ordering of the cells in $K$ 89. We deal here with a particular ordering based on a cover forest $T$ of $K$ (any two vertices are connected by exactly one path in $T$ if and only if they are connected in $K)$. Let $T=(V, E)$ be a cover forest of $K$ where $V$ is the set of all the vertices of $K$ and $E$ a subset of edges of $K . S=\left(\sigma_{0}, \ldots, \sigma_{m}\right)$ is a $T$-filter if it is an ordering of all the cells in $K$ such that:

- for each $j$ (where $0 \leq j \leq m$ ), $\left\{\sigma_{0}, \ldots, \sigma_{j}\right\}$ is a subcomplex of $K$;

- if $i<j$, the dimension of $\sigma_{i}$ is less or equal than the dimension of $\sigma_{j}$;

- if $i<j, \sigma_{i}$ and $\sigma_{j}$ are two edges and $\sigma_{j} \in T$, then $\sigma_{i} \in T$ That is, the edges of the cover forest are in first positions in $S$ ).

Observe that $\sigma_{0}$ is always a vertex of $K$. An AT-model for $K$ is then defined as the output of the following algorithm, having as the input a complex $K$ and a $T$-filter $S$ of $K$.

Algorithm 1. 89] AT-model Algorithm.

$$
\begin{aligned}
& \text { InPuT: a } T \text {-filter } S=\left(\sigma_{0}, \ldots, \sigma_{m}\right) \text { of } K, \\
& H:=\left\{\sigma_{0}\right\}, f\left(\sigma_{0}\right):=\sigma_{0}, g\left(\sigma_{0}\right):=\sigma_{0}, \phi\left(\sigma_{0}\right):=0 . \\
& \text { For } i=1 \text { to } m \text { do } \\
& \quad \text { If } f d\left(\sigma_{i}\right)=0 \text {, then } \\
& \quad H:=H \cup\left\{\sigma_{i}\right\}, f\left(\sigma_{i}\right):=\sigma_{i}, \phi\left(\sigma_{i}\right):=0, g\left(\sigma_{i}\right):=\sigma_{i}+\phi d\left(\sigma_{i}\right) . \\
& \text { If } f d\left(\sigma_{i}\right) \neq 0 \text {, then: } \\
& \quad k:=\max \left\{j \text { such that } \sigma_{j} \in f d\left(\sigma_{i}\right), j=1, \ldots, i-1\right\}, \\
& \quad H:=H \backslash\left\{\sigma_{k}\right\}, f\left(\sigma_{i}\right):=0, \phi\left(\sigma_{i}\right):=0 . \\
& \quad \text { For } j=1 \text { to } i-1 \text { do if } \sigma_{k} \in f\left(\sigma_{j}\right), \\
& \quad f\left(\sigma_{j}\right):=f\left(\sigma_{j}\right)+f d\left(\sigma_{i}\right), \phi\left(\sigma_{j}\right):=\phi\left(\sigma_{j}\right)+\sigma_{i}+\phi d\left(\sigma_{i}\right) .
\end{aligned}
$$

Output: the set $(S, H, f, g, \phi)$.

Notice that in the $i$ th step of the algorithm $(i=1, \ldots, m)$, exactly one homology generator is created or destroyed. The algorithm runs in time at most $\mathcal{O}\left(\mathrm{m}^{3}\right)$.

Proposition 1. Let $K$ be a complex (simplicial, cubical or simploidal), let $T=$ $(V, E)$ be a cover forest of $K$ and $S$ a T-filter of $K$. The output of Algorithm 1 , $(S, H, f, g, \phi)$, satisfies that:

- $H$ is a subset of $S$ such that no edge in $T$ is an edge in $H$. $H$ generates a chain complex denoted by $\mathcal{H}$ with null differential;

- The number of vertices, edges and triangles in $H$ equals the number of connected components, tunnels and cavities in $|K|$, respectively. In other words, the homology of $K$ is isomorphic to $\mathcal{H}$.

$-f: C(K) \rightarrow \mathcal{H}$ satisfies that if $c$ ad $c^{\prime}$ are two cycles in $K$ such that $f(c)=$ $f\left(c^{\prime}\right)$ then $c$ and $c^{\prime}$ are homologous. 
- $g: \mathcal{H} \rightarrow C(K)$ satisfies that $\{[g(h)]: h \in H\}$ is a set of homology generators of $K$. If $h, h^{\prime} \in H, h \neq h^{\prime}$, then $g(h)$ and $g\left(h^{\prime}\right)$ are not homologous. Moreover, if a is an edge in $H$, then $g(a)$ is a simple cycle in $K$ and all the edges in $g(a) \backslash\{a\}$ are edges in T. In fact, $g(a) \backslash\{a\}$ is the simple path in $T$ connecting the endvertices of $a$.

$-\phi: C(K) \rightarrow C(K)$ satisfies that if $x \in H$ then $\phi(x)=0$ and there is no $y \in S$ such that $\phi(y)=x$. Moreover, if $v$ is a vertex in $K$, then $\phi(v)$ is the simple path in $T$ connecting $v$ with the vertex in $H$ that belongs to the same connected component in $K$ than $v$.

$\overbrace{0}^{1}$\begin{tabular}{c|c|c|c|c|c|}
\hline$S$ & $H$ & $f$ & $g$ & $\phi$ \\
\hline$\langle 0\rangle$ & $\langle 0\rangle$ & $\langle 0\rangle$ & $\langle 0\rangle$ & 0 \\
$\langle 1\rangle$ & & $\langle 0\rangle$ & & $\langle 0,1\rangle$ \\
$\langle 2\rangle$ & & $\langle 0\rangle$ & & $\langle 0,2\rangle$ \\
$\langle 0,1\rangle$ & & 0 & & 0 \\
$\langle 0,2\rangle$ & & 0 & & 0 \\
$\langle 1,2\rangle$ & $\langle 1,2\rangle$ & $\langle 1,2\rangle$ & $\langle 1,2\rangle+\langle 0,2\rangle+\langle 0,1\rangle$ & 0 \\
\hline
\end{tabular}

Fig. 3. A a filter $S$ of a simplicial complex $K$ and the result of applying Algorithm 1 to $S$ (an AT-model for $K$ )

\section{Computing a Graph-with-Loop Representation of a 3D Object}

Let $K$ be a complex (simplicial, cubical or simploidal); $|K|$ its geometric realization in $\mathbf{R}^{3}$; and $h:|K| \rightarrow \mathbf{R}$ a continuous function. Let $e_{x y}$ denote an edge with endvertices $x$ and $y$. We say that the height of a point $p \in|K|$ is $t$ if $h(p)=t$ and the height of a cell $\sigma \in K$ is the minimum of the heights of all the points on $|\sigma|$. We say that $K$ is an $h$-complex if:

- the set of the vertices of $K$ can be partitioned into a finite number of subsets in terms of their height, $V=\bigcup_{i=1}^{r} V_{i}$, where $V_{i}=\left\{v \in V: h(v)=t_{i}\right.$ and $t_{1}<\cdots<t_{r}$.

- if $e_{v w}$ is an edge in $K$ then $v$ and $w$ belong to $V_{i}$ for some $i=1, \ldots, r$ or $v \in V_{i-1}$ and $w \in V_{i}$ for some $i=2, \ldots, r$.

$h$-Complexes appear in a natural way when they are defined by the neighborhood relations of voxels of a 3D digital image and $h$ is the real function that associates to each point on $|K|$ its elevation.

Let $K$ be an $h$-complex and $\sigma$ a cell in $K$. We say that $\sigma$ is horizontal if the heights of all the points on $|\sigma|$ coincide; otherwise, it is vertical. For $i=1, \ldots, r$, let $K_{i}$ be the collection of all the horizontal cells in $K$ with the same height $t_{i}$, $i=0,1, \ldots, r . K_{i}$ is a subcomplex of $K$ and if a cell $\sigma$ is not in $K_{i}$, then $\sigma$ is vertical. Let $T_{i}=\left(V_{i}, E_{i}\right)$ be a cover forest of $K_{i}$ and $S_{i}$ a $T_{i}$-filter of $K_{i}$. Denote by $\left(S_{i}, H_{i}, f_{i}, g_{i}, \phi_{i}\right), i=1, \ldots, r$, the AT-models obtained using Algorithm 1 Let $V$ be the set of vertices in $K, T=(V, E)$ a cover forest of $K$ (obtained 

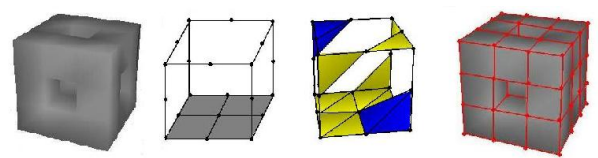

Fig. 4. From left to right: a digital image and $3 h$-complexes associated to it considering the 6,14 and 26-adjacency, respectively

after adding vertical edges in $K$ in increasing ordering in height to the graph $\left.\left(V, \bigcup_{i=1}^{r} E_{i}\right)\right)$, and $S$ a $T$-filter of $K$. Denote by $(S, H, f, g, \phi)$ the AT-model obtained using Algorithm 1

Proposition 2. The AT-model $(S, H, f, g, \phi)$ satisfies that:

- If $a$ is a horizontal edge in $H$, then $a \in H_{i}$ for some $i$ and $g(a)=g_{i}(a)$ is a simple cycle such that its edges are in $K_{i}$.

- If $a$ is a vertical edge in $H$, then for each level $i, i=1, \ldots, r, g(a)$ has an even number of vertical edges of height $t_{i}$.

Now, let us explain how to construct the graph $G_{h}(K)$ and the function $\Psi$ : $G_{h}(K) \rightarrow K$ using the AT-models $\left(S_{i}, H_{i}, f_{i}, g_{i}, \phi_{i}\right), i=1, \ldots, r$ and $(S, H, f, g, \phi)$ computed before. First, the vertices in $G_{h}(K)$ in each level $i$ are the vertices in $H_{i}$, $i=1, \ldots, r$. If $v$ is a vertex in $G_{h}(K)$, then $\Psi(v)=v$. Second, for each level $i$ and (horizontal) edge $a$ in $H_{i} \cap H$, we add a loop $\alpha$ in $G_{h}(K)$ such that its endvertex is the vertex in the level $i$ of $G_{h}(K)$ which belongs to the same connected component than $|a|$ in $\left|K_{i}\right|$. Define $\Psi(\alpha)=g_{i}(a)=g(a)$. Third, we add an edge $e_{x y}$ between two vertices $x$ and $y$ in $G_{h}(K)$ if $x \in H_{i}$ and $y \in H_{i+1}$ for some $i$ and $f(x)=$ $f(y)=z \in H$ (i.e. $x$ and $y$ belong to the same connected component in $K$ ). Define $\Psi\left(e_{x y}\right)=\phi(x)+\phi(y)$ (the simple path in $T$ connecting the vertices $x$ and $y$ ). Finally, for each vertical edge $e_{v w}$ in $H$, an edge $b$ is added to $G_{h}(K)$. Since $e_{v w}$ is vertical, then $v \in H_{i}$ and $w \in H_{i+1}$ for some $i$. The endvertices of $b$ are the vertices in $G_{h}(K)$ which belong to the same connected component than $v$ in $K_{i}$ and $w$ in $K_{i+1}$, respectively. Define $\Psi(b)=e_{v w}+\phi_{i}(v)+\phi_{i+1}(w)$.

Theorem 2. Given a complex $K$ and a continuous function $h:|K| \rightarrow \mathbf{R}$. If $K$ is an h-complex, then:

1. The graph $G_{h}(K)$ and the complex $K$ has the same number of tunnels and connected components.

2. For each loop $\alpha \in G_{h}(K), \Psi(\alpha)$ is a simple cycle representative of a homology generator of $K$. If $\alpha_{1}$ and $\alpha_{2}$ are two different loops in $G_{h}(K)$, then $\Psi\left(\alpha_{1}\right)$ and $\Psi\left(\alpha_{2}\right)$ are two representative cycles of two non-equivalent generators of homology.

3. For each edge $e_{x y}$ in $G_{h}(K)$ that comes from a vertical edge $e_{v w} \in H$, then $\Psi\left(e_{x y}\right)+\phi(x)+\phi(y)=g\left(e_{v w}\right)$ is a representative cycle of a homology generator of $K$.

4. The graph $G_{h}(K)$ without loops is a subdivision of the Reeb graph $R_{h}(K)$.

5. The graph $G_{h}(K)$ and the function $\Psi$ can be computed in $\mathcal{O}\left(m^{3}\right)$, where $m$ is the number of cells in $K$. 
Proof. The number of tunnels of $K$ is the number of edges in $H$. By construction, each horizontal edge in $H$ produces a loop in $G_{h}(K)$ (i.e. a tunnel in $G_{h}(K)$ ). Each vertical edge $e_{v w}$ in $H$ produces a vertical edge $\beta$ in $G_{h}(K)$. Let $v$ in $K_{i}$ and $w$ in $K_{i+1}$. Let $V$ and $W$ be the two vertices in $G_{h}(K)$ that belong to the same connected component than $v$ and $w$, respectively. Since $e_{v w} \in H$, then $e_{v w}$ created a cycle when it was added. Therefore, $v$ and $w$ belong to the same connected component in $K$ and so, there exists a path $p$ between $V$ and $W$ in $G_{h}(K)$ apart from the edge $\beta$ that produces $e_{v w}$, by construction. Then, $p+\beta$ is a cycle in $G_{h}(K)$. Moreover, $\Psi(p+\beta)=g(b)$ is a representative cycle of the homology generators of dimension 1 of $K$. Since representative cycles of a homology generator of dimension 1 of $K$ map by $\psi$ to a cycle in $R_{h}(K)$, then $\psi(g(b))$ is a cycle in $R_{h}(K)$. Since $K$ is an $h$-complex, then a vertex in $R_{h}(K)$ corresponds to a contour in a level $t_{i}$, $i=1, \ldots, r$. Therefore, a vertex in $R_{h}(K)$ is a vertex in $G_{h}(K)$.
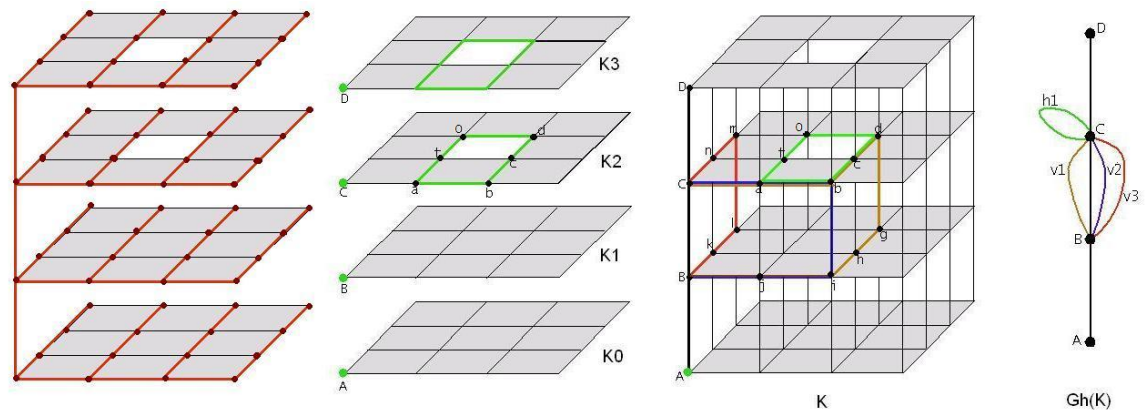

Fig. 5. From left to right: a cover forest $T$ of the cubical complex $K$ showed on the right of Figure 4; the complexes $K_{0}, K_{1}, K_{2}$ and $K_{3}$; a set of representative cycles of the generators $H_{1}(K)$; and the graph $G_{h}(K)$

Example 1. Let $K$ be the cubical complex $K$ on the right in Figure 4. A cover forest $T$ of $K$; the complexes $K_{0}, K_{1}, K_{2}$ and $K_{3}$; a set of representative cycles of the generators $H_{1}(K)$; and the graph $G_{h}(K)$ The non-trivial identification of the edges and loops in $G_{h}(K)$ and $K$ by $\Psi$ are:

\begin{tabular}{c|c}
$G_{h}(K)$ & $\Psi$ \\
\hline$h_{1}$ & $e_{a b}+e_{b c}+e_{c d}+e_{d o}+e_{o f}+e_{a f}$ \\
$v_{1}$ & $e_{B j}+e_{i j}+e_{h i}+e_{g h}+e_{d g}+e_{c d}+e_{a c}+e_{a b}+e_{b C}$ \\
$v_{2}$ & $e_{B j}+e_{i j}+e_{b i}+e_{a b}+e_{a C}$ \\
$v_{3}$ & $e_{B k}+e_{\ell k}+e_{m \ell}+e_{m n}+e_{n C}$
\end{tabular}

The representative cycles of generators of $H_{1}(K)$ are:

$$
\begin{aligned}
& \alpha_{0}=\Psi\left(h_{1}\right)=e_{a b}+e_{b c}+e_{c d}+e_{d o}+e_{o f}+e_{a f} \\
& \alpha_{1}=\Psi\left(v_{1}\right)+e_{B C}=e_{B j}+e_{i j}+e_{h i}+e_{g h}+e_{d g}+e_{c d}+e_{a c}+e_{a b}+e_{b C}+e_{B C} \\
& \alpha_{2}=\Psi\left(v_{2}\right)+e_{B C}=e_{B j}+e_{i j}+e_{b i}+e_{a b}+e_{a C}+e_{B C} \\
& \alpha_{3}=\Psi\left(v_{3}\right)+e_{B C}=e_{B k}+e_{\ell k}+e_{m \ell}+e_{m n}+e_{n C}+e_{B C}
\end{aligned}
$$




\section{Conclusions and Future Work}

It is possible to obtain representative cycles on the boundary of the given complex $K$ if we compute a cover forest of $K$ first adding the edges on the boundary. Another task is the generalization of the method to any dimension. The problem is that the homology of a complex of a dimension higher than 3 can have torsion groups. In order to capture the torsion part of the homology we could use the concept of $\lambda$-AT-model developed in [10].

A possible extension of this work is the construction of a discrete Morse complex $M_{h}(K)$ associated to a cell complex $K$, such that there is not only a oneby-one identification of all the homology generators of $M_{h}(K)$ with that of $K$, but also an isomorphism between cohomology rings. $M_{h}(K)$ can be constructed using a gradient vector field $\mathcal{V}_{K}$ associated to a discrete Morse function (see [6] that can be obtained from an AT-model for $K$.

\section{References}

1. Alexandroff, P., Hopf, H.: Topologie I. Springer, Berlin (1935)

2. Biasotti, S., Facidieno, B., Spagnuolo, M.: Extended Reeb Graphs for Surface Understanding and Description. In: Nyström, I., Sanniti di Baja, G., Borgefors, G. (eds.) DGCI 2000. LNCS, vol. 1953, pp. 185-197. Springer, Heidelberg (2000)

3. Dahmen, W., Micchelli, C.A.: On the Linear Independence of Multivariate bSplines. Triangulation of Simploids. SIAM J. Numer. Anal., 19 (1982)

4. Cole-McLaughlin, K., Edelsbruner, H., Harer, J., Natarajan, V., Pascucci, V.: Loops in Reeb Graphs of 2-mainifolds. Discrete Comput. Geom. 32, 231-244 (2004)

5. Fomenko, A.T., Kunii, T.L.: Topological Methods for Visualization. Springer, Heidelberg (1997)

6. Forman, R.: A discrete Morse theory for cell complexes. In: Yau, S.T.(ed.) Geometry, Topology and Physics for Raoul Bott. International Press (1995)

7. Forman, R.: Discrete Morse Theory and the Cohomology Ring. Transactions of the American Mathematical Society 354, 5063-5085 (2002)

8. Gonzalez-Diaz, R., Real, P.: Towards Digital Cohomology. In: Nyström, I., Sanniti di Baja, G., Svensson, S. (eds.) DGCI 2003. LNCS, vol. 2886, pp. 92-101. Springer, Heidelberg (2003)

9. Gonzalez-Diaz, R., Real, P.: On the Cohomology of 3D Digital Images. Discrete Applied Math. 147, 245-263 (2005)

10. Gonzalez-Diaz, R., Jiménez, M.J., Medrano, B., Real, P.: Extending AT-Models for Integer Homology Computation. In: GbR2007. LNCS, vol. 4538, pp. 330-339. Springer, Heidelberg (2007)

11. Massey, W.M.: A Basic Course in Algebraic Topology. New York (1991)

12. Munkres, J.R.: Elements of Algebraic Topology. Addison-Wesley, London, UK (1984)

13. Reeb, G.: Sur les Points Singuliers d'une Forme de Pfaff Complement Integrable ou d'une Function Numérique. C. Rendud Acad. Sciences 222, 847-849 (1946) 